\title{
Exponential MLWDF (EXP-MLWDF) Downlink Scheduling Algorithm Evaluated in LTE for High Mobility and Dense Area Scenario
}

\author{
Ismail Angri $^{1}$, Mohammed Mahfoudi ${ }^{2}$, Abdellah Najid ${ }^{3}$, Moulhime El Bekkali ${ }^{4}$ \\ ${ }^{1,3}$ Telecommunication Systems, Networks and Services laboratory - National Institute of Posts and Telecommunications \\ (INPT) Rabat, Morocco \\ ${ }^{2,4}$ Transmission and Data Processing Laboratory (LTTI) - Superior School of Technology - Fez, Morocco
}

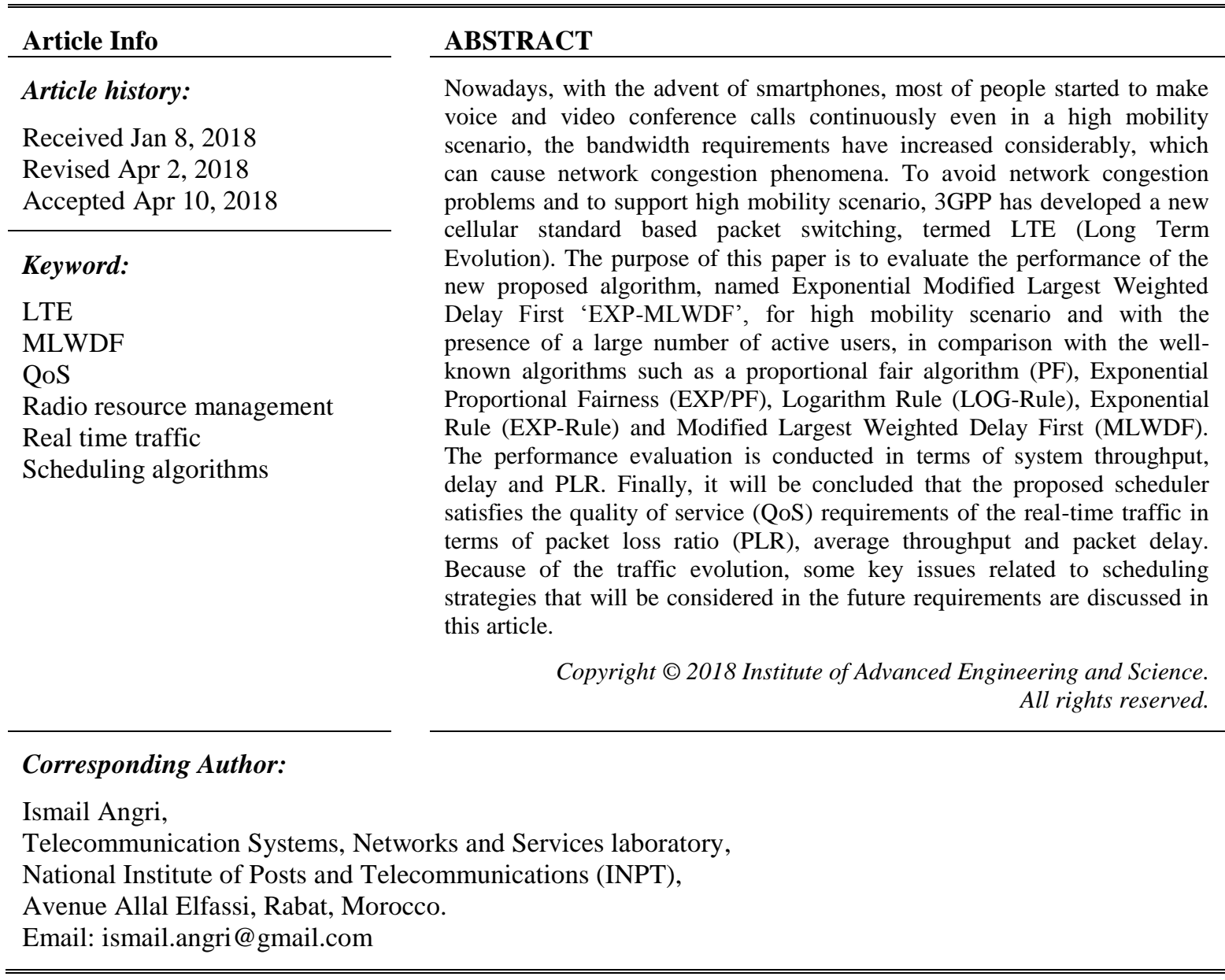

\section{INTRODUCTION}

Customer demand for new services in mobile networks, with high quality (QOS) increase from day to day. Due to this exponential increase, operators require a wider bandwidth, high efficiency and low cost [1], [2].

In a dense environment with high-speed UEs, radio resource management is becoming a big problem, so the use of an efficient scheduling algorithm will be mandatory [3].

Several factors influence the QoS of the LTE and LTE-A networks. Among them the transmission channel conditions, the type of services used and the number of radio resources available to each user. The concept of resource block (RB) is that adopted by LTE as a transmission unit. The RB is a block of subcarriers with a number of OFDM symbols, operating in both frequency and time domain [1]. 
Both TDD and FDD modes are used to operate an LTE network. As can be seen in Figure 1, the LTE frame in the FDD mode has duration of $10 \mathrm{~ms}$, divided into 10 subframes with duration of $1 \mathrm{~ms}$. Each subframe consists of two time slots of 500ns. A number of six or seven OFDM symbols represent a single slot [4].

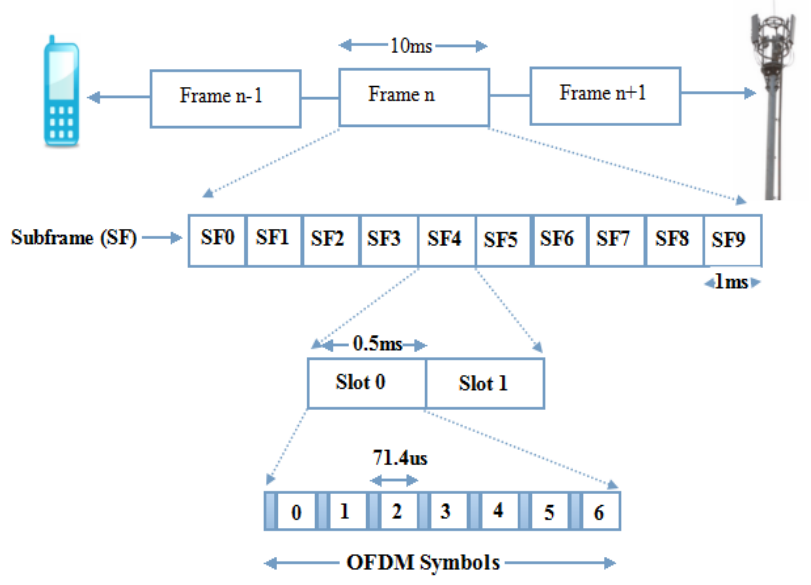

Figure 1. LTE Frame structure [5]

The objectives of LTE are to support high mobility and to avoid network congestion [6]. As in the case of uplinkbands [7], downlink scheduling algorithms must ensure a good sharing of existing radio resources, and especially in the case of real-time traffic (Video and VOIP streams) [8].

The purpose of this paper is to evaluate the performance of the new proposed algorithm in LTE system, named Exponential Modified Largest Weighted Delay First 'EXP-MLWDF', for high mobility scenario, with the presence of a large number of active users.

\section{SCHEDULING STRATEGIES}

The MAC (Medium Access Control) layer is the responsible layer at the base station of the scheduling process. In LTE, the separation between uplink and downlink scheduling decisions is assured, and the management of both types is independent. A parameter called CQI (Channel Quality Indicator) represents the status of the channel and gives information about its characteristics; this information is used by the scheduler for decision making [9]. At each time period called TTI (Transmission Time Interval), the CQI report is updated [10].

The scheduling strategies in uplink and downlink have been programmed separately in a Multicell/multi-user environment in the LTE-sim simulator. These models take into account all the real parameters that influences those decisions such as user mobility, frequency reuse techniques and radio resource management [11].

The new LTE network architecture defined by 3GPP is based on simplicity. The creation of a FullIP system was made with the aim of improving mainly the throughput and the transmission delay compared to the UMTS networks [12]. A dedicated frequency spectrum for the LTE network can be used to avoid problems of incompatibility with other wireless networks systems [13].

The main protocols configured in the eNb and used in the two levels Control and User Plane are illustrated in Figure 2, in addition to the different functions of the two layers 1 and 2 [14].

The protocol stack for the User Plane consists of multiple sub-layers: PDCP (Packet Data Convergence Protocol) which provides its services to the upper layers, among these services the transfer of user plane data, transfer of control plane data and header compression. RLC (Radio Link Control) used on the radio interface for reliable data transmission. MAC (Media Access Control) which is responsible for the access control to the medium and PHY (the physical layer) which is responsible for the effective transmission of the radio waves [15].

The protocol stack for the control Plane is composed on the PDCP, RLC, MAC, PHY ans RRC sublayers. The scheduling process and the CQI (Channel Quality Indicator) manager that receives the CQI reports (that are very useful for the scheduling decisions) for Downlink traffic are performed by those presented in protocol stack sublayers [16]. The MAC sub layers of the eNb have several objectives. They 
construct, send, receive and process transport blocks that contain a series of user-plane data from one or more bearers.

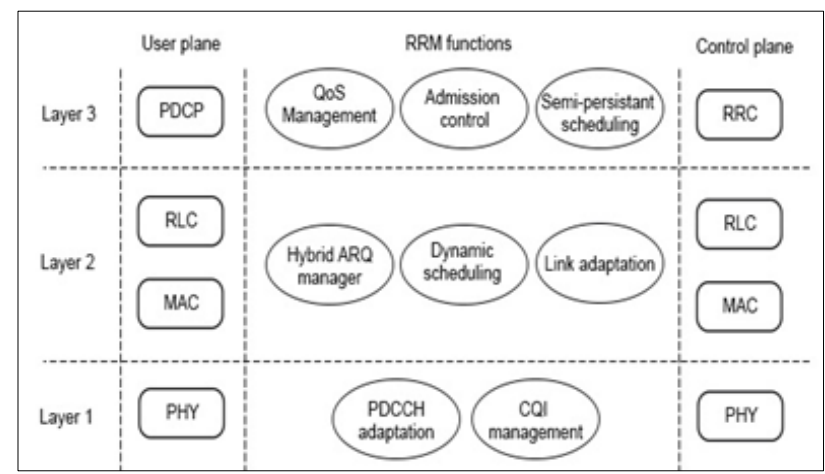

Figure 2. LTE Protocols Stack

In order to optimize the system performance and meet QoS client requirements, radio resources must be assigned to the customer in an efficient manner. And to do this, the MAC scheduler runs the scheduling algorithms that determine how the channels are used in the air interface and that in both downlink and Uplink modes. In Figure 3, a model representation of a simplified packet scheduler in the downlink LTE system is shown.

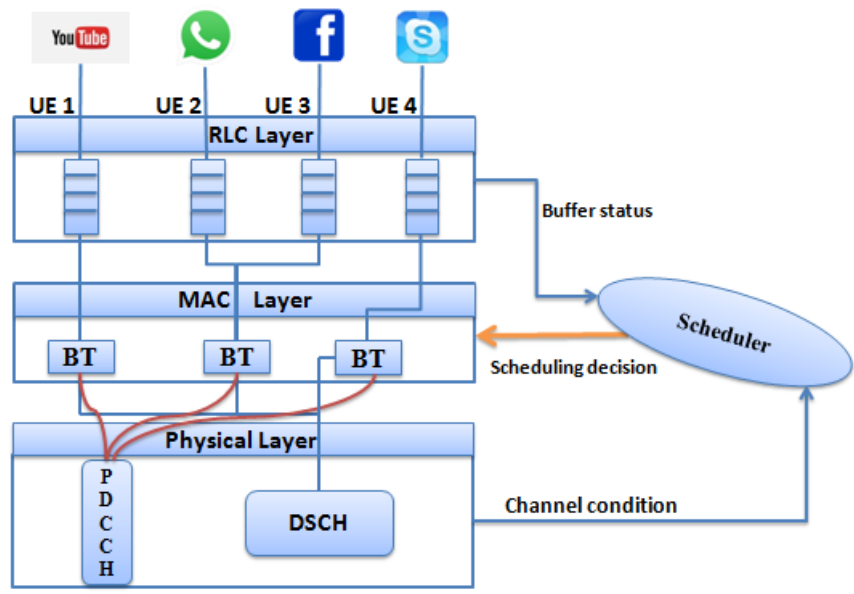

Figure 3. Packet Scheduling Model in LTE System

Different sequences of a specific scheduler are used to calculate the instantaneous metrics assigned to each separated stream. We assume that the metric assigned to stream i on $j$-th sub-channel is defined by $w i, j$. To calculate the metric by each scheduler, two main parameters are mandatory, namely: the available flow rate to the UE on the $j$-th sub-channel and the average transmission rate $\overline{\mathrm{R}}_{\mathrm{i}}$ of flow $\mathrm{i}$ [17].

In the next equation we give an estimation representation of $\overline{\mathrm{R}}_{\mathrm{i}}$. This value is calculated at each TTI using $\overline{\mathrm{R}}_{\mathrm{i}}(\mathrm{k}-1)$ as the average transmission data rate estimated at the $(k-1)$-th TTI and $\mathrm{R}_{\mathrm{i}}(\mathrm{k})$ is the rate allocated to $i$-th flow during the $k$-th TTI:

$$
\bar{R}_{i}(k)=0.8 \bar{R}_{i}(k-1)+0.2 R_{i}(k)[17]
$$


In the following, a summary description of three different scheduling algorithms that are used in all simulation scenarios, these are: PF as well as EXP-PF and MLWDF, In addition to other well-known algorithms.

\subsection{Proportional fair $(\mathbf{P F})$}

With the main objective of ensuring fairness between the different data streams transmitted to each UE and also to achieve a maximum total network throughput, the PF (Proportional Fair) scheduler was introduced to assign the radio resources to different users and this based on the characteristics of the channel as well as the history of the flows rate of the previous transmissions.

For this scheduler, We can define $r i, j$ by taking into consideration the CQI value on the $j$-th subchannel which is sent by the UE who is intended for $i$-th flow. This parameter is calculated using the AMC module. The metric is then the ratio between the instantaneous flow available for $i$-th flow and the medium flow that was calculated at the moment (k-1) [18], [19].

$$
w_{i, j}=\frac{r_{i, j}}{\overline{R_{I}}}
$$

\subsection{Exponential proportional fairness (EXP/PF)}

To promote the real time traffic, The EXP/PF algorithm was introduced. It requires the not exceeding of a defined delay threshold given the criticality of the real-time RT flows with respect to nonReal time NRT flows.Its main goal is the processing of the multimedia services, especially in an Adaptive Coding \& Modulation/Time Division Multiplexing (ACM/TDM) system [20].

For real time flows, the metric is calculated by using the following equations [20]:

$$
w_{i, j}=\exp \left(\frac{\alpha_{i} D_{H O L, i}-X}{1+\sqrt{X}}\right) \frac{r_{i, j}}{\overline{R_{l}}}
$$

Where $D_{H O L, i}$ is the Head of line Delay, and $X$ is given by [19]:

$$
X=\frac{1}{N_{r t}} \sum_{i=1}^{N_{r t}} \alpha_{i} D_{H O L, i}
$$

With $N_{r t}$ is the number of active real time flows in downlink direction.

\subsection{Logarithm rule (LOG-Rule)}

To give much more QoS support to the network [9]. The metric for the LOG rule is given as:

$$
\mathrm{w}_{\mathrm{i}, \mathrm{j}}^{\mathrm{LOG} \text { Rule }}=\mathrm{b}_{\mathrm{i}} \log \left(\mathrm{c}+\alpha_{\mathrm{i}} \mathrm{D}_{\mathrm{HOL}, \mathrm{i}}\right) \Gamma_{\mathrm{j}}^{\mathrm{i}}
$$

Where $\Gamma_{\mathrm{j}}^{\mathrm{i} S i g n i f i e s}$ the channels spectral efficiency with the $i$-th user at the $j$-th $\mathrm{RB}$, for $1 \leq \mathrm{i} \leq \mathrm{N}$. However it was found that to get much better performance [15], it's better to declare the constraints $\alpha \mathrm{i}$, bi, and $\mathrm{c}$ as follow:

$$
\alpha_{i}=\left[\frac{5}{\left(0.99 \alpha_{\mathrm{i}}\right)}\right], \mathrm{b}_{\mathrm{i}}=\left[\frac{1}{\mathrm{E}\left(\Gamma^{\mathrm{i}}\right)}\right], \mathrm{c}=1.1
$$
scheduling.

$\tau_{\mathrm{i}}$ in the previous equation represents the packet delay threshold for each RT flow at the moment of

\subsection{Exponential rule (EXP-Rule)}

To be more robust, and to have improvements in delay and throughput [15], The EXP rule scheduler metric is defined as follows [21], [22]:

$$
\mathrm{w}_{\mathrm{i}, \mathrm{j}}^{\text {EXP Rule }}=\mathrm{b}_{\mathrm{i}} \exp \left(\frac{\alpha_{\mathrm{i}} \mathrm{D}_{\mathrm{HOL}, \mathrm{i}}}{\mathrm{c}+\sqrt{\left(\frac{1}{\mathrm{~N}_{\mathrm{rt}}}\right) \Sigma_{\mathrm{i}} \mathrm{D}_{\mathrm{HOL}, \mathrm{i}}}}\right) \Gamma_{\mathrm{j}}^{\mathrm{i}}
$$


Where the following constraint's values are the same as Log Rule's parameters:

$$
\alpha_{i} \in\left[\frac{5}{\left(0.99 \tau_{i}\right)}, \frac{10}{\left(0.99 \tau_{i}\right)}\right], b_{i}=\left[\frac{1}{E\left(\Gamma^{i}\right)}\right], c=1
$$

\subsection{Modified largest weighted delay first (MLWDF)}

The classification of the streams according to their type (Real-time or not Real-time) is used by this algorithm to promote the real time flows which have the best propagation conditions and the highest time, in order to be transmitted at first in the canal. The M-LWDF metric is defined by [17]:

$$
w_{i, j}=\alpha_{i} D_{H O L, i} \frac{r_{i, j}}{\overline{R_{1}}}
$$

Where $r_{i, j}$ and $\bar{R}_{i}$ have the same signification as cited in Nomenclature chapter and $\alpha \mathrm{i}$ is given by:

$$
\alpha_{\mathrm{i}}=-\frac{\log \left(\delta_{\mathrm{i}}\right)}{\tau_{\mathrm{i}}}
$$

$\alpha \mathrm{i}$ is defined with considering $\tau$ as a time threshold package and $\delta$ as the maximum probability that the delay $D_{H O L, i}$ exceeds the threshold time [16].

\section{THE PROPOSED EXPONENTIAL MLWDF (EXP-MLWDF)}

The channel conditions play an important role in wireless communication systems to maximize the throughput obtained by the user. We define $r i, j$ as the current data rate that could be used by the $i$-th user on the $j$-th sub-channel at time $t$. Thus, users who have a better channel condition will get higher priority to send their packages. So we can propose the metric as shown below:

$$
w_{i, j}=r_{i, j}
$$

Therefore, we need to consider the users who don't have a good channel conditions. Thus, users will be punished. Hence the necessity to consider the estimated average transmission data rate at the $(k-1)$-th Transmission Time Interval (TTI). So the new metric must be:

$$
w_{i, j}=\frac{r_{i, j}}{\overline{R_{1}}}
$$

For each real-time data streams and similar to MLWDF algorithm, we define $\alpha_{\mathrm{i}}$ according to the Equation (10).

From the QoS information's sent to the eNodeB by the users we can get the Head of line Delay $D_{H O L, i}$ for the $i$-th user, which is an important parameter that we must take in consideration. Normally to further the users who don't have good channel conditions, the smaller the difference between the deadline delay and the time spend in queue $D_{H O L, i}$, the higher metric must be. This is explained by the term:

$$
\frac{\tau_{i}}{\left(\tau_{i}-D_{\text {HOL }, i}\right)}
$$

If we applied the exponential function to the previous term, the critical conditions can be served:

$$
\exp \left(\frac{\tau_{i}}{\left(\tau_{i}-D_{H O L, i}\right)}\right)
$$

When we combined all the cited parameters, we obtained the proposed algorithm as defined below:

$$
w_{i, j}=\frac{\left(\alpha_{i} * r_{i, j}\right)}{\overline{R_{1}}} \exp \left(\frac{\tau_{i}}{\left(\tau_{i}-D_{H O L, i}\right)}\right)
$$




\section{SIMULATION, RESULTS AND DISCUSSION}

The different performances of our proposed algorithm are tested in comparison with the other algorithms already existing. To do that, an open source tool named LTE-Sim was used. It is a simulator based on different classes programmed in $\mathrm{C}++$ to represent the models of all the techniques and components of a 4G network layers and sublayers. The different classes can be extended to meet the needs of each user [23].

The various aspects necessary for a real simulation of UpLink and DownLink scheduling strategies in a Multi-Cell/Multi-User environment have been well integrated into the software.

Before starting the simulation in the chosen tool, the definition of a real scenario is mandatory; its role is to fix the different parameters and characteristics to simulate. For example, in our case, a Single Cell with Interference scenario was adopted. Our environment contains 3 cells, each one have a radius of $1 \mathrm{Km}$. Users (which have a defined number between 10 and 100) moves randomly between the cells using the RANDOM_WALK mobility model with a high speed of $120 \mathrm{~km} / \mathrm{h}$. They are uniformly distributed in the three cells. The simulation results were established for the period of which the UEs move inside the cell. Each user receives an H.264 Video stream, a VoIP stream, and BE flows modeled by Infinite Buffer.

The purpose of this simulation is to evaluate the performance of our new proposed scheduling algorithm in LTE Network, for this we compared its performances with the well-known algorithms such as the PF, M-LWDF and EXP-PF schedulers [21], by measuring packet latency (the delay), Packet Loss Ratio (PLR) and packet throughput. The simulation parameters are illustrated in the following Table 1.

Table 1. Simulation Parameters

\begin{tabular}{cc}
\hline Parameters & Value \\
\hline Simulation duration & $100 \mathrm{~s}$ \\
Flows duration & $120 \mathrm{~s}$ \\
Frame structure & FDD \\
Mobile speed & $120 \mathrm{Km} / \mathrm{h}$ \\
Radius & $1 \mathrm{~km}$ \\
Bandwidth & $20 \mathrm{MHz}$ \\
Slot duration & $0.5 \mathrm{~s}$ \\
Scheduling time ( TTI duration) & $1 \mathrm{~ms}$ \\
Number of Resource Blocks (RBs) & 100 \\
Max delay & $0.1 \mathrm{~s}$ \\
Video bit-rate & $242 \mathrm{kbps}$ \\
VoIP bit-rate & $8.4 \mathrm{kbps}$ \\
Minimum number of users & 10 \\
Maximum number of users & 100 \\
Interval between users & 5 \\
Simulation duration & $100 \mathrm{~s}$ \\
\hline
\end{tabular}

\subsection{The measurement of packet latency (delay)}

Latency has a most noticeable influence on Network performance. Especially for conversational services, such as VoIP and Video Flows that require low latency [22]. Other services that benefit from low delay are gaming and applications with extensive handshaking, such as emails.

Latency and TTI (Transmission Time Interval) are two interrelated parameters. It is difficult to improve one of them without the degradation of the other.

\subsubsection{The VOIP flows}

The Figure 4 demonstrates that the proposed algorithm presents a very low delay and its stable even the increase of the user's number. The same behavior is observed for EXP-PF, EXP-Rule, LOG-Rule end MLWDF algorithms. For the PF algorithm, the delay shows highest value compared with the other algorithms by the increase of users starting with 45 UE's. 


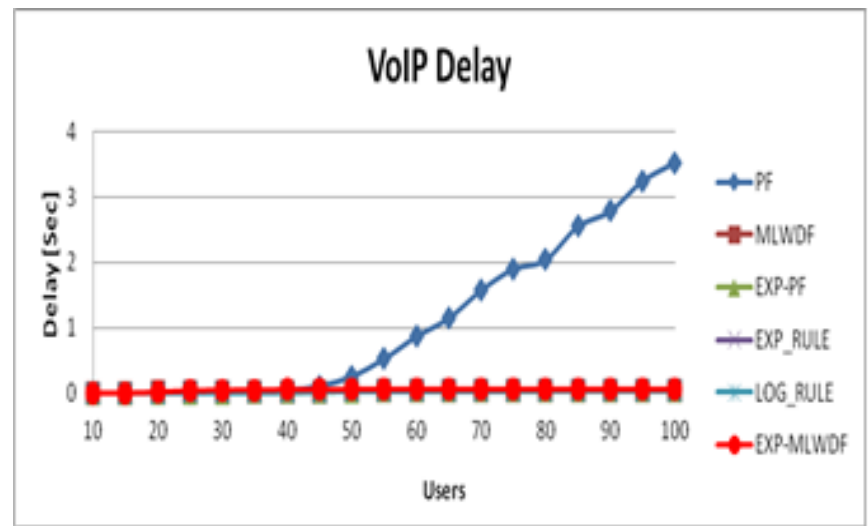

Figure 4. The Delay for VOIP Flows

\subsubsection{For video flows}

As we can see in Figure 5, The Video delay is very low for the EXP-MLWDF, our proposed scheduling algorithm, even the increment of user's number, comparing with $\mathrm{PF}$ and algorithms which presents a higher delay that increase exponentially with the increase of the user's number to get for 100 UE's $15 \mathrm{~s}$ which is not acceptable by the norms. The other algorithms present approximately the same behavior as our proposed algorithm.

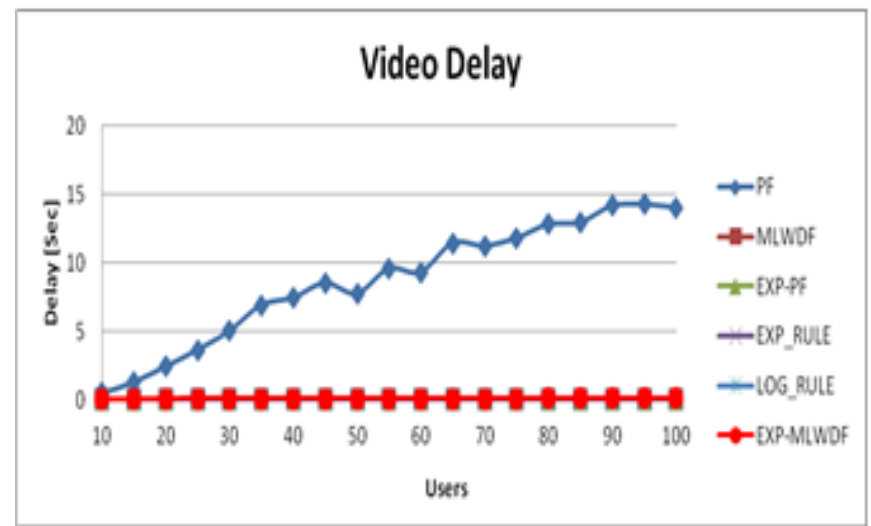

Figure 5. The Delay for Video Flows

\subsection{The measurement of packet loss ratio (PLR)}

The improvement of PLR estimation is a critical issue, because Packet Loss Ratio has a big effect on the network performance, especially when dealing with the real-time traffic such as VOIP and Video Flows [2].

\subsubsection{The VOIP flows}

The Packet Loss Ratio has given in Figure 6, it shows that our scheduling algorithm 'EXPMLWDF' presents a very low packet loss ratio that stay negligible until 50 UEs, starting from 50 UEs, our algorithm continues to present the low value compared with other algorithms in which the PLR starts to increase exponentially with the increase of the user's number, for 100 UE's EXP-MLWDF get very low value about $12 \%$ compared with others algorithms which get about $40 \%$. 


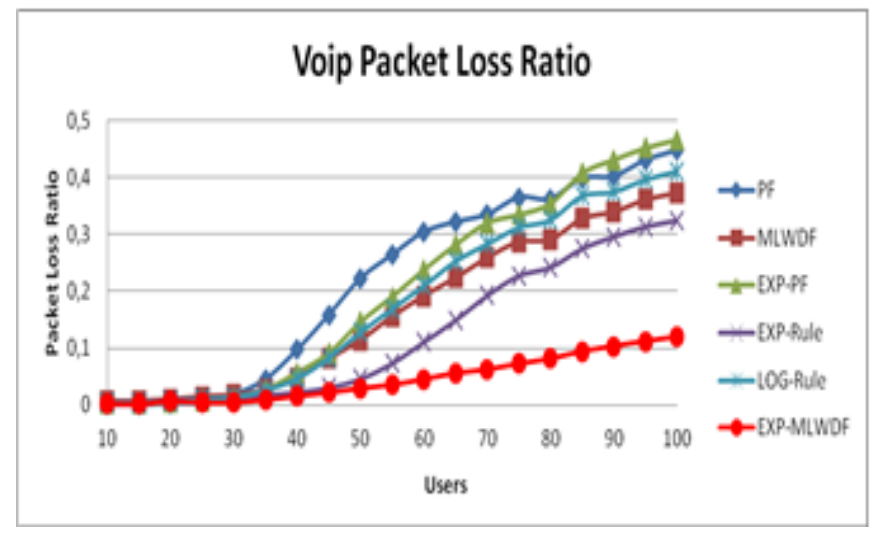

Figure 6. The Packet Loss Ratio (PLR) for VoIP Flow

\subsubsection{For video flows}

The Packet Loss Ratio for video flows is given on Figure 7, it is noticed that the PLR given by our proposed algorithm is the lowest comparing with other algorithms such as MLWDF, EXP/PF, LOG-Rule, EXP-Rule and especially PF who shows a dramatic increase by the increase of the user's number. In high mobility scenario and with the presence of a big number of users in the cell, the PLR value is very large, about $90 \%$ for all the compared algorithms with the distinction of our algorithm which continue to present the low PLR value.

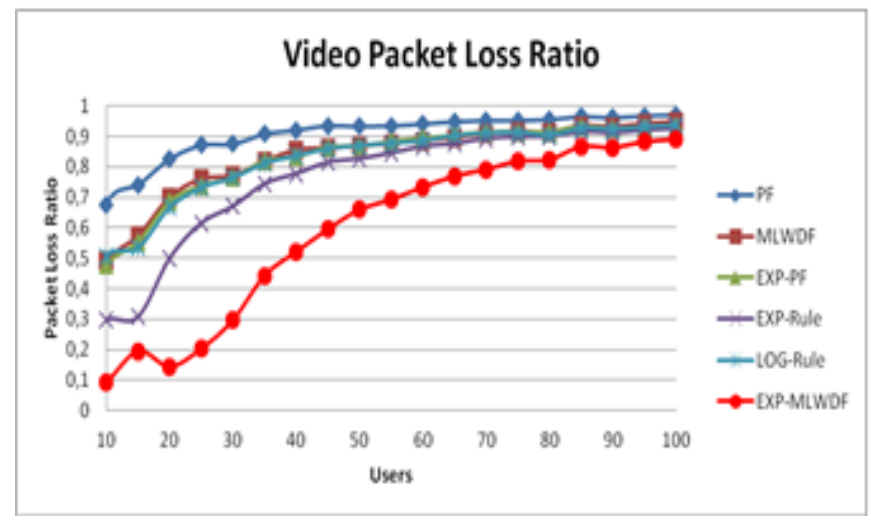

Figure 7. The Packet Loss Ratio (PLR) for Video Flow

\subsection{The measurement of packets throughput}

The Packets Throughput is measured on the basis of the average success rate of the different packets transmitted to the destination using the appropriate radio channel [2].

\subsubsection{For VOIP flows}

The packet throughput for VOIP flows increases exponentially with the number of users, and it's the same for all the scheduling algorithms, with the distinction of our proposed algorithm 'EXP-MLWDF' that presents a high throughput. The EXP-PF presents the lower throughput value (see Figure 8). 


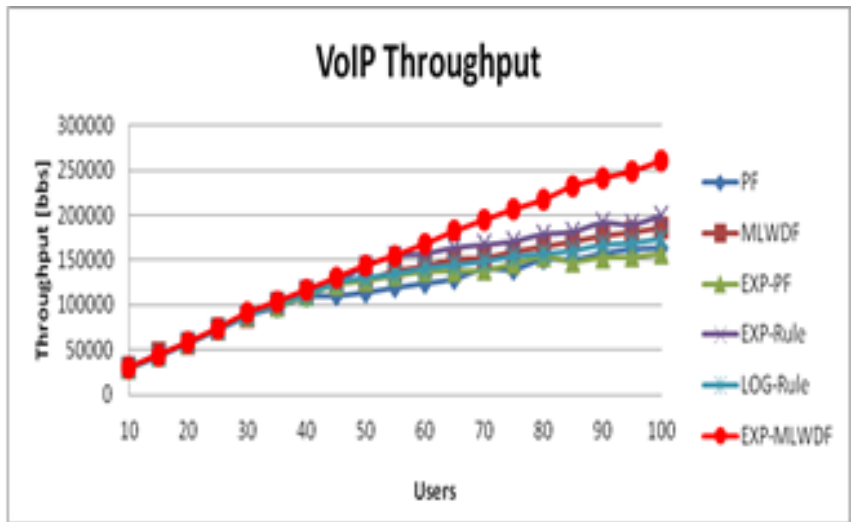

Figure 8. The packets throughput for VOIP Flows

\subsubsection{For video flows}

Figure 9 represents the Video packet throughput. It shows that the throughput for the entire scheduling algorithms increases as long as the number of users increases until 30 UEs. The proposed algorithm 'EXP-MLWDF' presents the very high throughput even the increase of the user's number, this throughput value starts to decrease starting from $30 \mathrm{UEs}$ from $1 \mathrm{Mbit} / \mathrm{s}$ to $600 \mathrm{Kbit} / \mathrm{s}$ for $100 \mathrm{UEs}$. The PF scheduler presents the very low throughput compared with other algorithms.

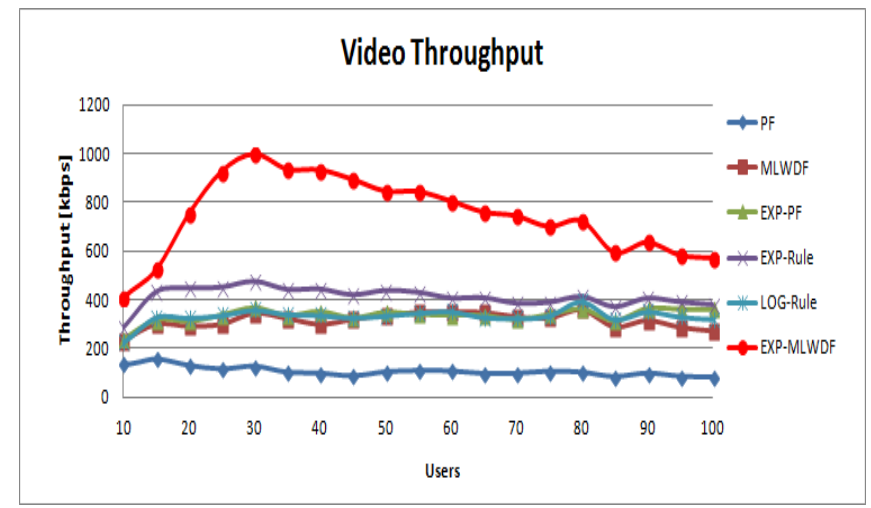

Figure 9. The packets throughput for Video Flows.

\section{CONCLUSION}

In this paper, our work focused on packet scheduling issues in the downlink of 3GPP LTE networks. We proposed a new algorithm named EXP-MLWDF, and we compared its performances with some wellknown algorithms, such as the proportional fairness algorithm (PF), Exponential Proportional Fairness (EXP/PF), the Modified Largest Weighted Delay First (MLWDF), the LOG-Rule and the EXP-Rule, for high mobility scenario with the presence of a big number of users. This comparison is conducted in terms of packet delay, Packet loss ratio 'PLR' and Throughput. Simulation results have confirmed that our algorithm is more advanced and satisfies QoS requirements of Real Time services. Our future work will be devoted to propose an algorithm for the Uplink pathway to achieve the best overall system performance.

\section{REFERENCES}

[1] 3GPP TS 32.423 version 10.8.0, Digital cellular telecommunications system; LTE, Release 10, April 2014.

[2] Iskandar I, Galih R, "Carrier Aggregation Technique to Improve Capacity in LTE-Advanced Network", TELKOMNIKA (Telecommunication, Computing, Electronics and Control), March 2016, vol. 14, no. 1, pp. 119-128.

[3] Iskandar I, Setyawan I, Nuraini H, "Inter-cell Interference Management Technique for Multi-Cell LTE-A Network", International Journal of Electrical and Computer Engineering (IJECE), 2017, vol. 7, no. 5. 
[4] Christopher C, "An Introduction to LTE: LTE, LTE-Advanced, SAE and 4G Mobile Communications", Wiley online library, First Edition, ISBN: 9781119970385, 2012, pp. 8-15.

[5] 3GPP, LTE; Evolved Universal Terrestrial Radio Access (E-UTRA); Physical channels and modulation, 3GPP TS 36.211, V12.3.0, 2014.

[6] Chen T, Zhao X, Gao T, Zhang L, "A novel downlink scheduling strategy for traffic communication system based on TD-LTE technology”, Springer Plus, 2016, vol. 5, no. 1.

[7] Shafinaz Bt I, Darmawaty Bt M, Norsuzila Y, "Performance Analysis of Uplink Scheduling Algorithms in LTE Networks", Indonesian Journal of Electrical Engineering and Computer Science (IJEECS), February 2018, vol. 9, no. 2 .

[8] Tarik G, Hadi L, Ali S, "QoE-aware Optimization of Video Stream Downlink Scheduling Over LTE Networks Using RNNs and Genetic Algorithm” Procedia Computer Science, 2016, vol. 94, pp. 232-239.

[9] Radhakrishnan S, Neduncheliyan S, Thyagharajan K.K. "A review of downlink packet scheduling algorithms for real time traffic in LTE-advanced networks", Indian Journal of Science \& Technology, January 2016, vol. 9, no. 4.

[10] Yuan-Ping L, Bin-Jie H, Hui Z, Zong-Heng W, Wei G, "A delay priority scheduling algorithm for downlink realtime traffic in LTE networks", Information Technology, Networking, Electronic and Automation Control Conference, IEEE, Chongqing, China, 2016.

[11] Piro G, LTE-Sim - the LTE simulator. [OnLine] Available: [http://telematics.poliba.it/LTE-Sim]

[12] Ibikunle F, Olowononi F, "Application of MIMO Technology to Systems Beyond 3G", International Journal of Informatics and Communication Technology, 2012, vol. 1, no. 2.

[13] 3GPP TS 23.002 V12.5.0, Digital cellular telecommunications system (Phase 2+); Universal Mobile Telecommunications System (UMTS); LTE; Network architecture, 2014.

[14] AngriI, Najid A, Mahfoudi M, "New Combined Downlink Scheduling Algorithm for LTE Networks", International Journal on Communications Antenna and Propagation (IRECAP), 2017, vol. 7, no. 1.

[15] Ole G, Andrea Z, Kashif M, Mattia C, Jawad R, Olav N. Ø., "Scheduling policies in time and frequency domains for LTE downlink channel: a performance comparison", IEEE Transactions on Vehicular Technology, 2017, vol. 66, no. 4.

[16] Meng-Shiuan P, Tzu-Ming L, Chun-Yuan C, Ching-Yen W., "Downlink traffic scheduling for LTE-A small cell networks with dual connectivity enhancement”, IEEE Communications Letters, April 2016, vol. 20, no. 4, vol. 796-799.

[17] 3GPP TR 25.913 V8.0.0, "LTE: Requirements for Evolved UTRA (E-UTRA) and Evolved UTRAN (E-UTRAN) (Release 8)", January 2009.

[18] Capozzi F, Piro G, Grieco L, Boggia G, Camarda P, "Downlink packet scheduling in lte cellular networks: Key design issues and a survey", IEEE Communications Surveys and Tutorials, 2012, vol. 15, no. 2, pp. 678-700.

[19] Rabie K.A, Mohamed H.A, Octavia A.D., "Performance analysis of Proportional Fair Scheduling in OFDMA Wireless Systems", 72nd IEEE Vehicular Tech. Conference Fall (VTC 2010-Fall), Ottawa, ON, Canada, 2010, pp. 6-9.

[20] Basukala R, Mohd Ramli H,Sandrasegaran K, "Performance Analysis of EXP/PF and M-LWDF in Downlink 3GPP LTE System", IEEE, First Asian Himalayas International Conference on Internet AH-ICI, Kathmandu, Nepal, 2009, pp. 1-5.

[21] Biswapratapsingh S, "Performance Comparison of packet scheduling algorithms for video traffic in LTE cellular network", International Journal of Mobile Network Communications \& Telematics (IJMNCT), June 2013, vol. 3, no. 3, pp. 9-18.

[22] Mahfoudi M, El Bekkali M, Mazer S, El Ghazi M, Najid A, "LTE network capacity analysis to avoid congestion for real time traffic", 14th Mediterranean Microwave Symposium (MMS) conference, Marrakech, Morocco, 2014.

[23] Piro G, Grieco L, Boggia G, Capozzi F, Camarda P, "Simulating LTE cellular systems: an open source framework", IEEE Trans. Veh. Technol., Oct 2010, vol. 60, no. 2, pp. 498-513.

\section{BIOGRAPHIES OF AUTHORS}

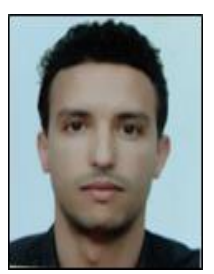

Angri Ismail was born in Akka, Tata, Morocco, on December 19, 1989. He received the Electronics and Telecommunication Engineering degree in 2013, from the Faculty of Sciences and Technics (FST), Sidi Mohamed Ibn Abdellah University, Fez, Morocco. Angri Ismail works as a Telecommunications Engineer at the National Telecommunications Regulatory Agency (ANRT), Rabat, Morocco. PhD student at the National Institute of Posts and Telecommunications, Rabat, Morocco. Email: Ismail.angri@gmail.com

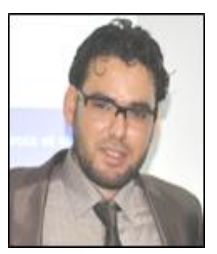

Mahfoudi Mohammed born in 1986 in Sefrou (Morocco), obtained graduate engineer degree in Telecommunications and Networks in 2010 at the National School of Applied Sciences of Fez. Mahfoudi Mohamed worked as Technical support engineer at Huawei Technology, Currently received the $\mathrm{PhD}$ in Telecommunications from Sidi Mohamed Ben Abdellah University Fez. Email: mahfoudi.mohammed@gmail.com 


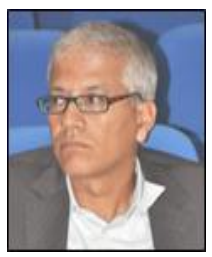

Najid Abdellah received the PhD. Degree in electronic engineering and the MS degree in Networking and Communication Systems from the ENSEEIHT of Toulouse, France; He has several years of research experiences with ENSEEIHT, ENSTA and INRIA. Dr. Abdellah Najid joined INPT RABAT Morocco as full professor of Microwave and Telecommunications engineering in 2000. He has devoted over 16 years to teaching Microwave engineering; Wireless Networking, Network Architecture, Network modeling courses and directing research projects in Wireless Network performance analysis; Wireless sensor Networking; Microwave and Antennas design. E-mail: najid@inpt.ac.ma

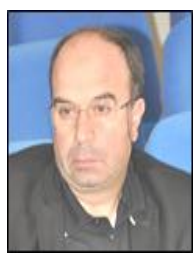

El Bekkali Moulhime received the PhD. Degree in 1991 from the USTL University - Lille 1 France, he worked in X-band printed antennas and their applications to microwave radar. He is professor in the Electrical Engineering Departement of the Superior School of Technology, Fez (ESTF). He is member of Transmission and Data Processing Laboratory (LTTI). Since 2009, Pr. Elbekkali served as vice President at University of Sidi Mohamed Ben Abdellah (USMBA) Fez, Morocco. 